\title{
Improving Dynamic Crowd Simulation using Visual Cues
}

\author{
Iznora Aini Zolkifly ${ }^{1^{*}}$, Abdullah Bade ${ }^{2}$ \\ ${ }^{1}$ Faculty of Business and Technology, UNITAR International University,3-01A, Level 2, Tierra Crest, \\ Jalan SS6/3, Kelana Jaya,47301 Petaling Jaya, Selangor Darul Ehsan, Malaysia \\ ${ }^{2}$ Faculty of Science and Natural Resources, Universiti Malaysia Sabah, Jalan UMS,88400, \\ Kota Kinabalu, Sabah, Malaysia
}

\begin{abstract}
Crowd simulations are used to predict dangerous situations during crowded events such as festivals, or to estimate if a sports stadium can be evacuated within a certain amount of time. During emergency incidents or escape panics situations, crowds are getting nervous and tend to do blind actions. Panicking crowds can be chaotic, irregular and unpredictable. Pushing, arching, clogging, jamming happens at exits. Most of the visual cues such as signage or exit marker has been ignored during panic or evacuation situation. This paper will explore the importance of visual cues in dynamic crowd simulation. It is also to discover the agent's responses to visual cues may influence navigational choices in emergent, panic or evacuation situation thus allow us to provide better situational awareness navigational aids in the virtual environment.
\end{abstract}

Keywords: crowd simulation; visual cues; agent's response

\section{INTRODUCTION}

Crowd simulations are used to predict dangerous situations during crowded events such as festivals, or to estimate if a sports stadium can be evacuated within a certain amount of time. In dynamic crowd simulation such as in emergent or evacuation situation, agents will perceive the environment to find a way out. At the beginning, most agents prefer nearer exit. However, some of the rooms or hall have multiple exits that made the agents faced a problem to decide the exit choice. The decision is influenced by several factors. When abrupt obstacles occur, agents must make decisions either to react fast to avoid the collision or do adjustable path planning to achieve goal with collision free path. Thus, collision prediction is important to develop solutions for real-time path finding to be able to react and readjust to any abrupt obstacles. The prediction methods consider the objects or agent's behaviours including state, velocity, goal and perception in the dynamic crowd simulation.

\section{MATERIALS AND METHODS}

Visual information plays an important role for agents to plan their movements adaptively. According to Moriarty (2004), visual cue is a signal and reminder of something, aiming to be self-explanatory. Some of the unfamiliar environments have insufficient navigation guides and confusing cues. Thus, it is important to have a navigation aids or visual cues to enhance the knowledge based of the virtual environment. Ant and honeybees use panorama skylines and visual panorama as their visual cues. Rats follow auditory cues to understand their environment (Rossier, et al., 2000). In robotics, visual cues can be detected through colours, edges, shape, texture, and motion. The need for the navigation aids in virtual environments (VEs) occurs for three reasons (Lawton,1994; Vinson,1999). The reasons are many VEs require the user 
to navigate, navigation in VEs is difficult, and disorientation is upsetting. Navigating unfamiliar environments can cause a problem. Visual cues usage in VEs allows further understanding of how human perform in real world environment. Agents need to perceive for the visual cues to observe urgency, importance, or uncertainty to decide which path to exit.

Visual cues influence on navigation in virtual environments. It affects perceptions and behaviour, and task performance on agents' vision. There are five types of elements in people's cognitive maps which are paths, edges, districts, nodes and landmarks (Lynch,1960). Landmarks that are noticeable and visible help navigators or agents remember their positions in the environment. Visual aids or cues must be clear and easy to understand. There are also visual cues in visual notification include digital signage, email notification, flat panel and message board displays. Visual cue works as directional cues for drawing attention to the areas of importance, such as interact with the environment.

According to Duarte et al., (2014), static, dynamic and memorized directional information are three different types of directional information. Signs or cues can be static and dynamic. (Bodeetal.,2014). Signs like arrow can catch people attention. However, there are some misleading and confusing visual cues. Figure 1 shows the example of confusing and misleading signage or cues.

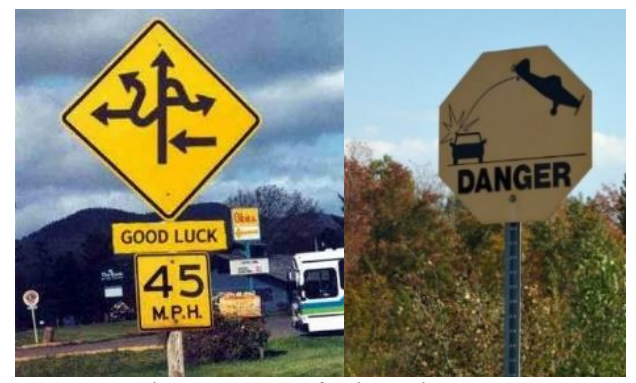

Figure 1. Confusing signage

Panic and chaos easily happen during emergency or evacuation situation. Emergencies situation may include fires, explosions, floods, earthquakes, storms, poisonous substantial leakages, workplace accidents and violence. Signs or cues are easier to interpret when people are not distracted by other. However, during panic or emergent situation, people are getting nervous and tend to make an unpredictable decision. Most of the visual cues and confusing signs has been ignored during panic or evacuation situation. Collective phenomena such as freezing by heating, transition to incoordination due to clogging, faster is slower effect due to impatience, phantom panics and ignorance of available exits may happen in panic situation.(Helbing,2002)Jams, arching and clogging happens at exits, fallen and injured people become obstacles. Herding behaviour may occur in exit path. Some of the people tend to follow other people (leader following) to find alternative exits. In mass behaviour, confused people will follow someone else. Therefore, visual cues or navigation aids are important to be clear and self-explain. Assembly points are well-labelled and designated with visual cues. With a good visual cues or signs, lives can be saved, and the chaos can be reduced. Visual safety cues such as universally known emergency imagery or maps can also inform non-native language speakers about any dangers. Figure 2 shows the scenario of dynamic crowd evacuation with abrupt obstacles. Figure 3 shows the examples of signage or cues.

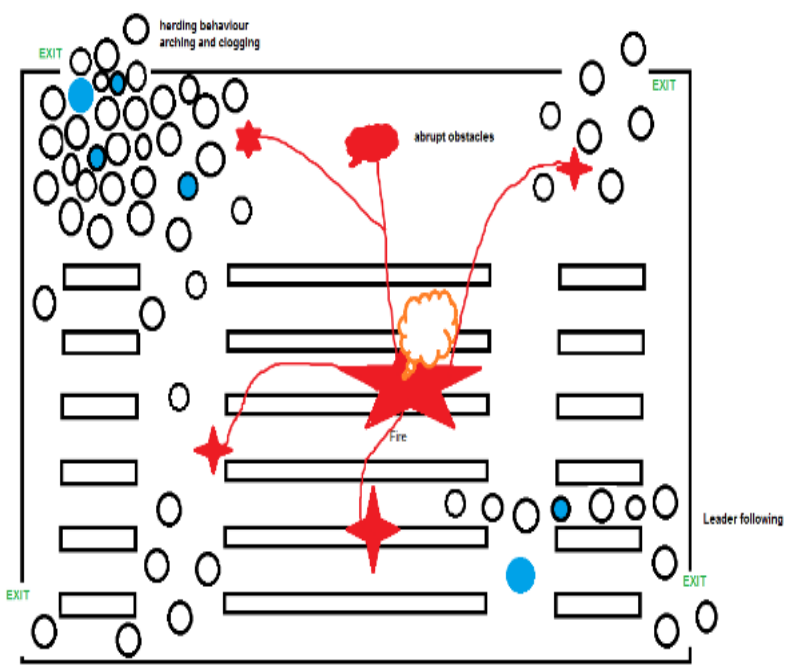

Figure 2. Crowd evacuation
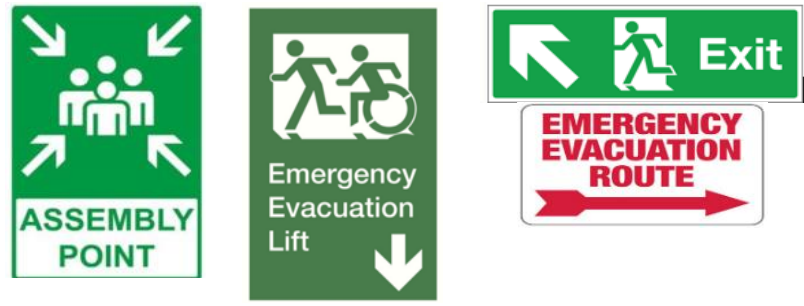

Figure 3. Signage

Another great way to provide visual cue on the floors especially as paths for emergency situation is footsteps marker with a glow-in-the-dark(Stop-painting,2017). In aviation safety, most of the flight has floor proximity lighting that aids airplane evacuation under dark or smoky 
conditions. (Anderson,2011). Figure 4 and 5 shows the example of footsteps marker and floor proximity lighting.

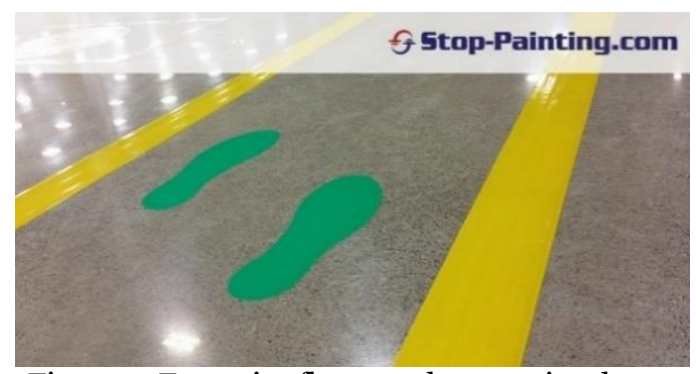

Figure 4. Footprint floor markers as visual cues

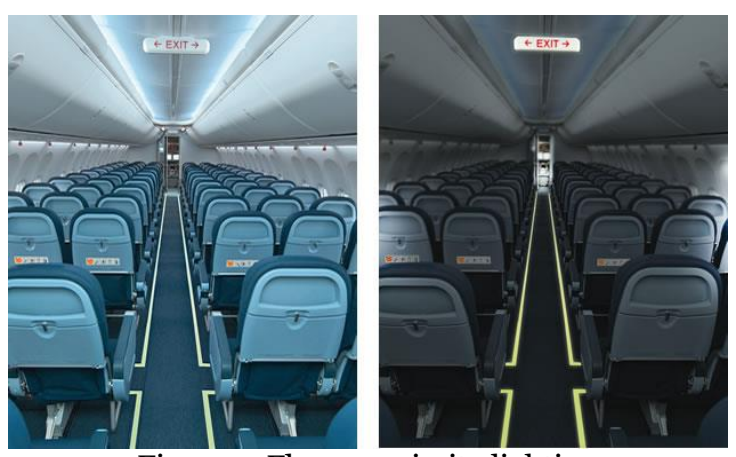

Figure 5. Floor proximity lighting

This crowd simulation will use Agent Based Modelling (ABM). Basically, agents will perceive the environment. If there is an event of emergency or evacuation, agents will check for visual cues (exit signs) and follow the cues to exit. If there are no visual cues, agents will check for the nearest exit according to the perceived information and the agent's radius of vision. The choice of the exit is based on the prior knowledge of the agents. While moving, agents constantly need to query the environment in order to avoid colliding with obstacles (Shao and Terzopolous,2005).

\section{RESULTS AND DISCUSSIONS}

This study was inspired by the work of Platynotan (2014), which presented the agent-based modelling (ABM) simulation of the Castello Ursino museum in Catania (Italy). The goal is to find out the importance of visual cues in crowd evacuation. There are ten rooms and three internal exits to the courtyard. There is only one main entrance/exit door at the museum. A series of tests are performed with different exit configuration. According to Pluchino et al., (2014), the number of 200 visitors is good to represent the real carrying capacity of the museum. Therefore, for the testing, the maximum number of agents will be 200 .
The test will compare the evacuation time for 1 exit, 2 exits and 3 exits without visual cues and with visual cues. The performance measure will be the evacuation time based on the tests below:

Test 1: Crowd simulation exit without visual cues

Test 2: Crowd simulation exit with visual cues

Test 1 scenario is to determine the time span needed for the agent's path finding to escape when no visual cue or exit direction signs are available. The agent's path is not predetermined, and each agent has a radius of vision. In this simulation, the number of agents tested is 100 and 200

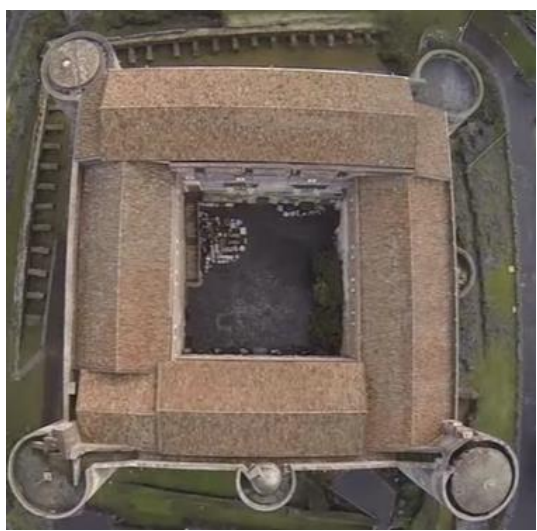

Figure 6. Castello Ursino museum

Figure 6 shows the Castello Ursino museum from the view of Stratos Mororad (2018). Figure 7 below shows the floor plan with 3 exits for the simulation in Netlogo.

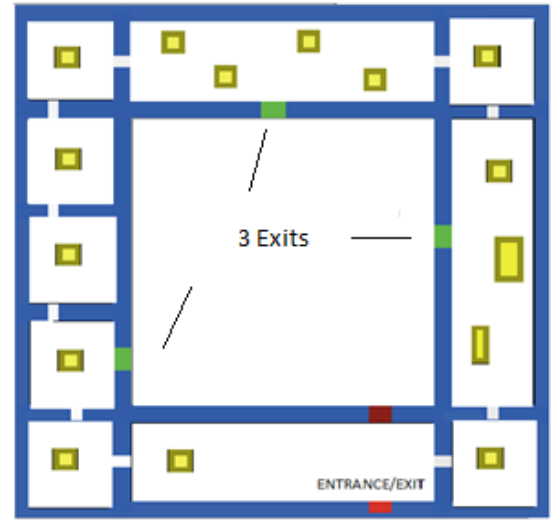

Figure 7. Floor plan for simulation in Netlogo

\section{A. Result of test simulations}

Testing was conducted for 100 agents and 200 agents. The same test case was repeated for five different runs. Basically, when emergency happen, agents will check if there is an exit within their radius vision. If yes, he moves 
towards the direction. If not, he will follow the same path he came from until the exit door is found. If there are visual cues present and visible to him, he will follow the cues.

Analysis for Test 1:

No of agents: 100, 200

Environment: Exits with no visual cues. Five test runs had been conducted for all exits without visual cues. The result for 100 agents is as stated in table 1 and figure 8 below:

Table 1: Escape times per second for all exits without visual cues for 100 agents

\begin{tabular}{|c|c|c|c|}
\hline \multirow{2}{*}{$\begin{array}{l}\text { No of } \\
\text { test }\end{array}$} & \multicolumn{3}{|c|}{ Escape time per seconds } \\
\cline { 2 - 4 } & 1 Exit & 2 Exits & 3 Exits \\
\hline 1 & 160 & 155 & 135.9 \\
\hline 2 & 156 & 155 & 137.9 \\
\hline 3 & 159 & 153.8 & 135.9 \\
\hline 4 & 162 & 157.1 & 135.3 \\
\hline 5 & 155.6 & 156.3 & 137.2 \\
\hline Average & 158.52 & 155.44 & 136.44 \\
\hline
\end{tabular}

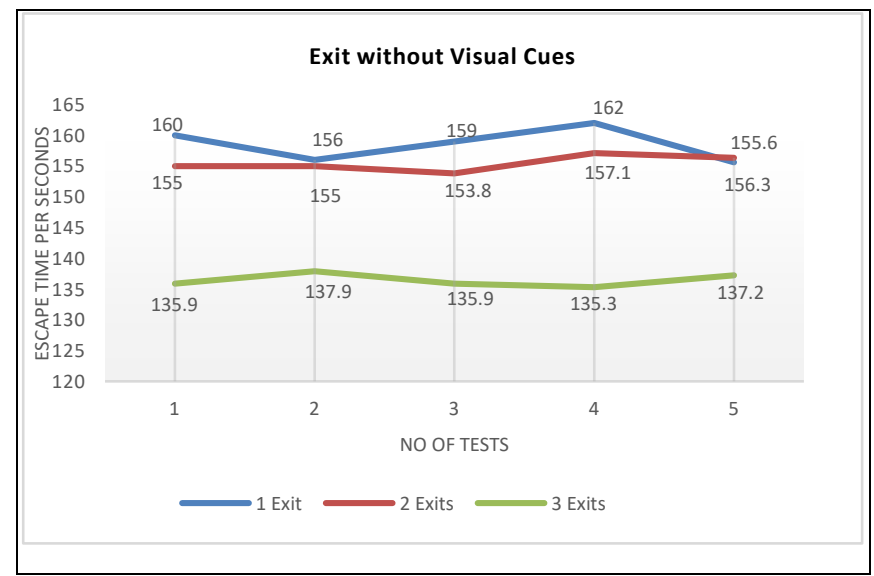

Figure 8. Exits without visual cues for 100 agents

Five test runs were conducted for all exits without visual cues for 200 agents. The result is as stated in table 2 and figure 9 below:
Table 2: Escape times per second for all exits without visual cues for 200 agents

\begin{tabular}{|c|c|c|c|}
\hline \multirow{2}{*}{ No of test } & \multicolumn{3}{|c|}{ Escape time per seconds } \\
\cline { 2 - 4 } & 1 exit & 2 exits & 3 exits \\
\hline 1 & 185.6 & 168 & 158.8 \\
\hline 2 & 186.4 & 164.5 & 159.9 \\
\hline 3 & 189.1 & 168.5 & 158 \\
\hline 4 & 185.6 & 163.2 & 157.2 \\
\hline 5 & 184 & 163.5 & 157.1 \\
\hline Average & 186.14 & 165.54 & 158.2 \\
\hline
\end{tabular}

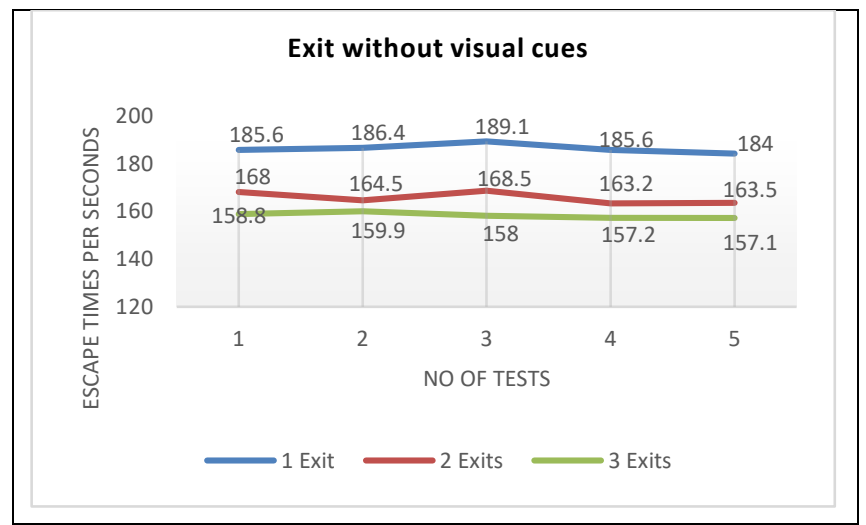

Figure. 9. Exits without visual cues for 200 agents

For both 100 agents and 200 agents, result for 1 exit ,2 exits, and 3 exits shows that the escape time for the agents in crowd evacuation are different. The escape time for 3 exits are faster compared to the 1 exit and 2 exits. It shows that more exits help the agents to escape the environment quicker. It also reduces the average value of evacuation time.

Analysis for Test 2:

No of agents: 100,200

Environment: Exits with visual cues. 


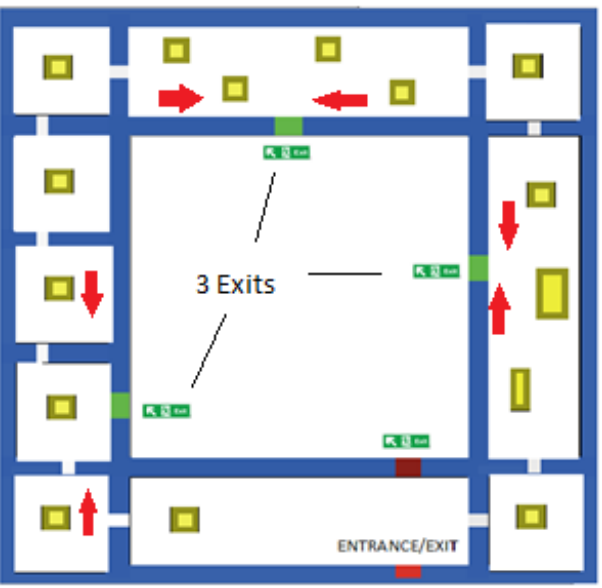

Figure 10: 3 Exits with visual cues

Figure 10 shows the exits with visual cues in Netlogo. Five test runs were conducted for all exits with visual cues for 100 agents. In this simulation, the visual cues are represented by arrows. The result is as stated in table 3 and figure 11 below:

Table 3: Escape times per second for all exits with visual cues for 100 agents

\begin{tabular}{|c|c|c|c|}
\hline \multirow{2}{*}{$\begin{array}{c}\text { No of } \\
\text { test }\end{array}$} & \multicolumn{3}{|c|}{ Escape time per seconds } \\
\cline { 2 - 4 } & 1 exit & 2 exits & 3 exits \\
\hline 1 & 137.2 & 125.6 & 123.2 \\
\hline 2 & 131.3 & 126 & 121.3 \\
\hline 3 & 130.8 & 124.2 & 121.8 \\
\hline 4 & 132.3 & 125.5 & 122.3 \\
\hline 5 & 133.1 & 125.7 & 121.2 \\
\hline Average & 132.94 & 125.4 & 121.96 \\
\hline
\end{tabular}

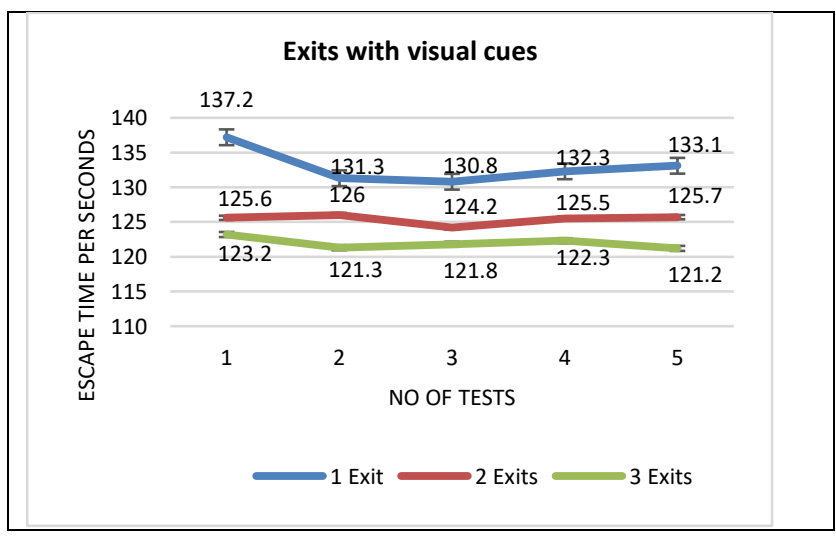

Figure 11. Exits with visual cues for 100 agents

Five test runs had been conducted for all exits without visual cues for 200 agents. The result is as stated in table 4 and figure 12.

Table 4: Escape times per second for all exits with visual cues for 200 agents

\begin{tabular}{|c|c|c|c|}
\hline \multirow{2}{*}{$\begin{array}{l}\text { No of } \\
\text { test }\end{array}$} & \multicolumn{3}{|c|}{ Escape time per seconds } \\
\cline { 2 - 4 } & 1 exit & 2 exits & 3 exits \\
\hline 1 & 172.3 & 157.3 & 151.2 \\
\hline 2 & 169 & 158.9 & 152.1 \\
\hline 3 & 171.9 & 158.4 & 152.3 \\
\hline 4 & 171.6 & 158.3 & 151.9 \\
\hline 5 & 172.8 & 159.9 & 152.2 \\
\hline Average & 171.52 & 158.56 & 151.94 \\
\hline
\end{tabular}

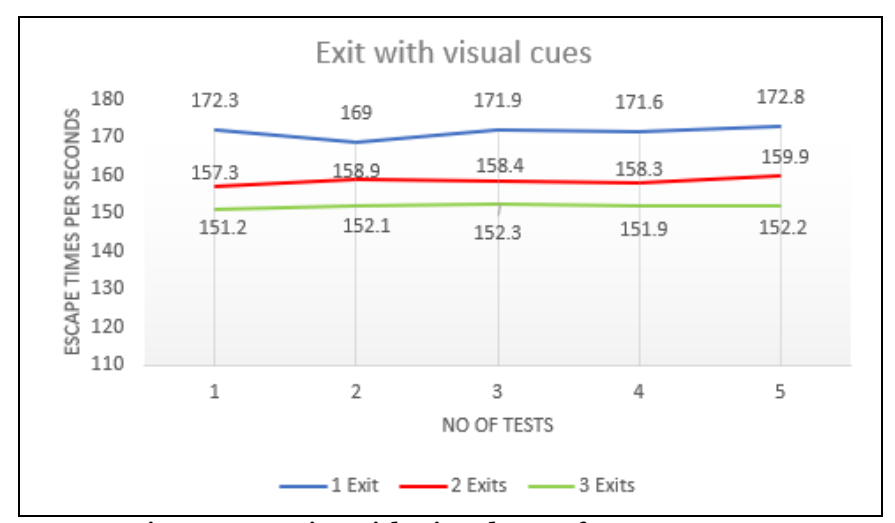

Figure 12. Exits with visual cues for 200 agents

Results for 1 exit, 2 exits and 3 exits show that the escape time for the agents in crowd evacuation with visual cues are faster compared to the environment without visual cues (Figure 8 and 9). Agents take longer time to find the way out without cues or signs. It shows that visual cues help and guide the agents to the exit. It also reduces the average value of evacuation time. The result also shows that when the agents saw the visual cues, he will follow the cues and reducing their evacuation time instead of coming back to the entrance.

\section{SUMMARY}

This paper explored the importance of visual cues in dynamic crowd simulation. Table 5 and Figure 13 shows the result summary for 100 agents.

Table 5: Escape times per seconds for visual cues and without visual cues for 100 agents

\begin{tabular}{|c|c|c|c|}
\hline $\begin{array}{c}\text { No of } \\
\text { exit }\end{array}$ & No visual cues & $\begin{array}{c}\text { With visual } \\
\text { cues }\end{array}$ & $\%$ \\
\hline 1 & 158.52 & 132.94 & $(16.14)$ \\
\hline 2 & 155.44 & 125.4 & $(19.33)$ \\
\hline 3 & 136.44 & 121.96 & $(10.61)$ \\
\hline
\end{tabular}




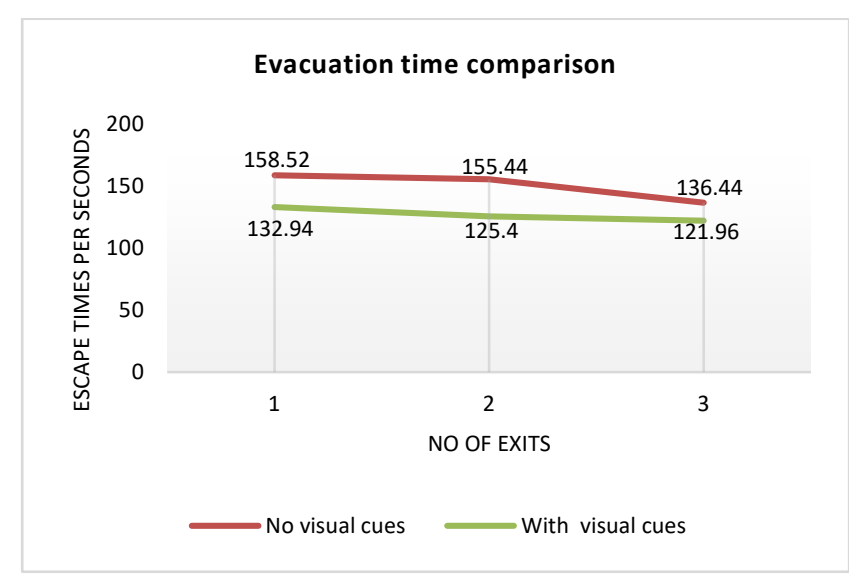

Figure 13. Exits with visual cues and no visual cues for 100 agents

Table 6 and Figure 14 shows the result summary for 200 agents.

Table 6: Escape times per seconds for visual cues and without visual cues 200 agents

\begin{tabular}{|c|c|c|c|}
\hline No of exit & $\begin{array}{c}\text { No visual } \\
\text { cues }\end{array}$ & $\begin{array}{c}\text { With } \\
\text { visual } \\
\text { cues }\end{array}$ & $\%$ \\
\hline 1 & 186.14 & 171.52 & $(7.85)$ \\
\hline 2 & 165.54 & 158.56 & $(4.27)$ \\
\hline 3 & 158.2 & 151.94 & $(3.95)$ \\
\hline
\end{tabular}

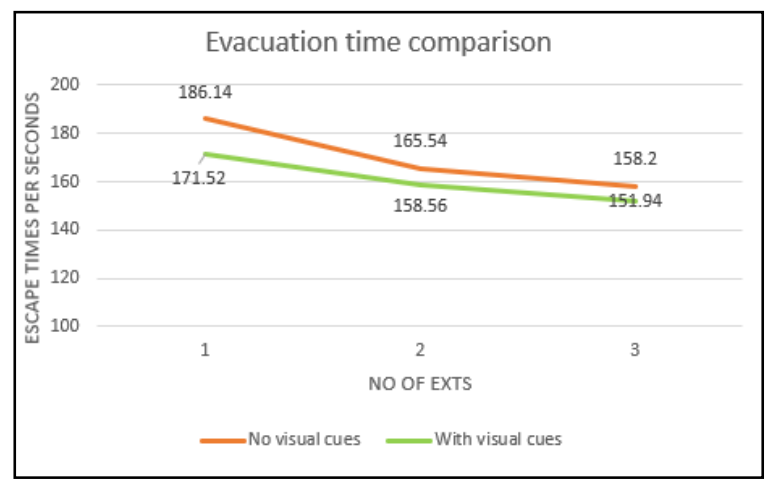

Figure 14. Exits with visual cues and no visual cues for 200 agents

Based on the results of the simulation, visual cues did help to reduce the escape times for agents in crowd evacuation. The number of exits also contributes to decrease the escape time. The visual cues or signage guide agents in making navigational choices to go to the exit door quicker.

The present of visual cues contributes more rapid exits compare to the absence of any visual cues results a slower exit. Thus, it improved the dynamic crowd simulation for emergent cases or evacuation. It is hoped that a good visual cues or signs help to provide better situational awareness navigational aids in the virtual environment.

\section{ACKNOWLEDGEMENT}

The author thanks the anonymous reviewer for their comments and UNITAR International University for the financial support. 


\section{REFERENCES}

Anderson, A. J. ,2011, Aviation Safety: evolution of airplane interiors. Retrieved from Boeng: Anderson, A. J. Aviation Safety: evolution of airplane interiors, viewed 20 August 2018<http://www.boeing.com/commercial/aeromagazi ne/articles/2011_q4/2/>

Bode, N. W. F., Kemloh Wagoum, A. U., \& Codling,2014, E. A., Human responses to multiple sources of directional information in virtual crowd evacuations. Journal of the Royal Society Interface,11(91), 20130904. http://doi.org/10.1098/rsif.2013.0904

Duarte, E., Rebelo, F., Teles, J., \& Wogalter, M. S.,2014, Behavioral compliance for dynamic versus static signs in an immersive virtual environment. Applied ergonomics, 45(5), pp 1367-1375.

Helbing, Dirk \& Farkas, Illés \& Molnar, Peter \& Vicsek, Tamás. 2002, Simulation of pedestrian crowds in normal and evacuation situations, pp.21-58.

Jérôme Rossier, Christian Haeberli, Françoise Schenk, 2000,"Auditory cues support place navigation in rats when associated with a visual cue," Behavioural Brain Research, vol. 117, no. 1-2, pp. 209-214.

Lawton, C. A. ,1994, Gender differences in way-findings strategies: Relationship to spatial ability and spatial anxiety. Sex Roles, 30(11-12), pp.765-779.

Lynch, Kevin .1960, The Image of the City. The MIT Press. ISBN 0-262-62001-4

Moriarty, S. E. November 2004. " An Interpretive Study of Visual Cues in Advertising," viewed 11 September 2018, <http://spot.colorado.edu/ moriarts/viscueing.html>

Pluchino A, Garofalo C, Inturri G, Rapisarda, A and Ignaccolo M, 2014, “Agent-Based Simulation of Pedestrian Behaviour in Closed Spaces: A Museum Case Study", Journal of Artificial Societies and Social Simulation ,17 (1) 16.,(doi: 10.18564/jasss.2336)
Stop-painting. 20 June 2017." Using Footprint Floor Markers as Visual Cues", viewed 10 July 2018, $<$ https://blog.stop-painting.com/using-footprintfloor-markers-visual-cues/>

Stratos M. and Castello, U., viewed 20 September2018<https://www.youtube.com/watch?v $=$ QU89JLHFW98>

Vinson, N. G., 1999, Design guidelines for landmarks to support navigation in virtual environments. In Proceedings of the SIGCHI conference on Human Factors in Computing Systems, pp. 278-285. ACM.

Wei Shao and Demetri Terzopoulos. 2005. Autonomous pedestrians. In Proceedings of the $2005 \mathrm{ACM}$ SIGGRAPH/Eurographics symposium on Computer animation (SCA '05). ACM, New York, NY, USA, 1928. DOI: https://doi.org/10.1145/1073368.1073371 